\title{
Cognitive Radio with Single Carrier TDCS and Multicarrier OFDM Approach with V-BLAST Receiver in Rayleigh Fading Channel
}

\author{
I. Budiarjo • H. Nikookar • L. P. Ligthart
}

Published online: 8 July 2008

(C) The Author(s) 2008

\begin{abstract}
This article presents the performance comparison of TDCS and OFDM based cognitive radio for MIMO system using VBLAST receiver architecture to reconstruct the transmitted data. The interference avoidance performance in terms of BER and bitrate are improved by adding multiple antennas to the system and the use of V-BLAST technique at the receiver. The results show the most promising interference avoidance technique combined with MIMO V-BLAST architecture to be applied in the CR system.
\end{abstract}

Keywords TDCS · OFDM · MIMO $\cdot$ V-BLAST .

Rayleigh Fading

\section{Introduction}

The scarcity of the spectrum resources for new wireless services and the spectral measurement results that indicate not all the time the spectrum is being used [1] have raised the development of cognitive radio (CR) technique. A CR

\footnotetext{
I. Budiarjo $(\bowtie) \cdot$ H. Nikookar $\cdot$ L. P. Ligthart

International Research Centre for Telecommunications,

Transmission and Radar (IRCTR),

Department of Electrical Engineering,

Mathematics, and Computer Science,

Delft University of Technology,

Mekelweg 4,

2628 CD Delft, The Netherlands

e-mail: i.budiarjo@irctr.tudelft.nl

H. Nikookar

e-mail: h.nikookar@irctr.tudelft.nl

L. P. Ligthart

e-mail: 1.p.ligthart@irctr.tudelft.nl
}

device utilizes the spectrum efficiently by learning and adapting its system to the environment condition [2].

A CR user is termed as a rental user (RU). An RU device has the capability to scan the spectrum and identify the region where the spectrum is occupied. A licensed user (LU) has the access priority to the spectrum hence the spectrum access by the RUs must not harm the LU's communications. This can be achieved by avoiding the spectrum usage on the region occupied by the LUs. Ortogonal frequency division multiplexing (OFDM) with carriers deactivation and windowing as interference avoidance technique in CR system has been proposed in [3], while in [4] adaptive bit loading is added to the OFDM system to obtain the target rate intelligently while the BER performance is kept preserved. Performance of adaptive bit loading with $2 \times 1-D$ Wiener filter channel estimation is evaluated in [5]. In [6] single carrier method using Fourier transform scheme with interference avoidance capability is introduced. The method is called "transform domain communication system" (TDCS).

This article shows the comparison between the two techniques in terms of computational complexity, BER and bitrate. Multiple input multiple output (MIMO) system with vertical Bell Laboratories Layered Space-Time (V-BLAST) architecture is added while research has shown that the capacity with this technique in a rich scattering environment is high [7]. Adding MIMO to the system will increase the spectral efficiency. OFDM is well known as a spectrally efficient modulation mode which due to its overlapped carriers spectrum but still orthogonal to each other, gives a higher bit rate compared to the single carrier transmission. As MIMO approach can fulfil the bit rate requirement for single carrier modulation, the BER performances of single carrier and multicarrier modulation need to be compared. 
This article is organized as follows. Section 2 describes the TDCS architecture in transmitter and receiver including its interference avoidance technique. OFDM scheme with spectrum pooling as interference avoidance method is given in Section 3. V-BLAST algorithm and the implementation model in TDCS and OFDM scheme are described in Section 4. Section 5 shows and compares the simulation results of TDCS and OFDM using V-BLAST in Rayleigh Fading channel model. The article is concluded in Section 6.

\section{Transform domain communication system (TDCS)}

The transmitter architecture of TDCS system is depicted in Fig. 1. TDCS is a single carrier transmission where its bandwidth can be divided into smaller subbands. In this way it is easier to locate the part of the band occupied by the LUs then not to put energy on that region. Information regarding the spectrum occupancy of the licensed users are distributed to each $\mathrm{CR}$ device. In our project the information is transmitted through common control channel [8]. Upon receiving the information about the region of the LU's band, a vector map $\boldsymbol{A}=\left[A_{0}, A_{1}, . ., A_{l}, \ldots, A_{N F F T-1}\right]$ is produced by Spectrum Magnitude where $l$ defines the index of subband. Zeros are placed on the subband indexes where the LU's band is located, and ones are placed on the other subband indexes. Guard interval (GI) is added to combat the multipath fading channel effect. Windowing can be added in order to reduce the sidelobes of the transmitted signal on the LU's band. TDCS gives more degree of freedom in choosing window to lower the sidelobes of its spectrum. Unlike in OFDM, the spectrum of the window of TDCS does not have to hit zero on the subband spacing interval as long as its sidelobes are very low, e.g. half sine window [9]. Inter subband interference will not degrade the data detection at the receiver due to the robustness of autocorrelation method. Windowing can be replaced by deactivating more subbands adjacent to the LU's band (adding more zeros to the vector $\boldsymbol{A}^{\prime}$ at the sub-bands adjacent to LU's band) since it will not reduce the transmission bit rate.

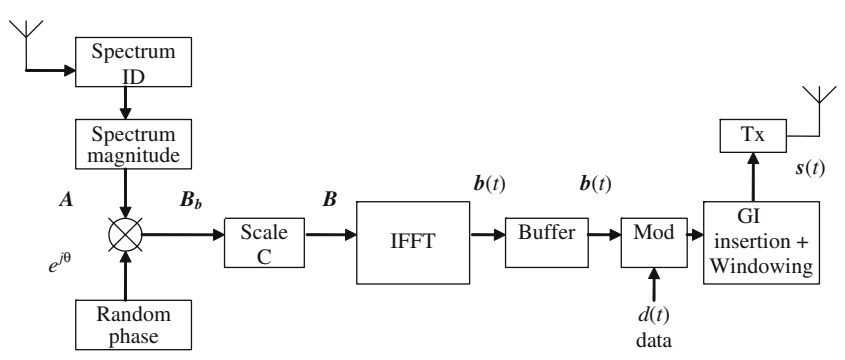

Fig. 1 Transmitter architecture of TDCS [5]
The impact of this zeros insertion can be described from the power density spectrum (PDS) equation,

$\operatorname{PSD}(f)=\frac{1}{N_{F F T}}\left|\sum_{l=0}^{N_{F F T-1}} A_{l} e^{j \theta_{l}} \int_{-(1+\alpha) \frac{T}{2}}^{(1+\alpha) \frac{T}{2}} p(t) e^{-j 2 \pi\left(f-f_{l}\right) t_{d t}}\right|^{2}$

where $T$ is the useful signal duration, $p(t)$ is the window function, $\alpha$ is the roll off factor of the window, and $\theta_{l}$ is the phase on subband $l$ produced by the random phase module. If $p(t)$ is a rectangular window and $\alpha$ is zero, the area within the integral can be replaced by $T \sin c\left(\pi\left(f-f_{l}\right) T\right)$. Zero amplitude at the carrier $l$ will make the PDS on that carrier position becoming zero, and due to the orthogonality among carriers the power contributions from the other carriers are also zero. The time domain signal $b(t)$ is combined with the transmitted data $d(t)$ using pulse amplitude modulation (PAM) or cyclic code shift keying (CCSK) scheme. In binary PAM the bits are mapped as the binary signals are the negative of each other, while in binary CCSK the binary signals are the cyclic shift by half of the symbol period of each other. A symbol is represented by $N_{\mathrm{FFT}}$ samples, accordingly the bit rate of TDCS system can be calculated as given in Eq. 2,

$R_{\mathrm{TDCS}}=\frac{\log _{2} \mathrm{CS}}{T_{s}^{\prime}}$,

where CS is the constellation size of modulation and $T_{\mathrm{S}^{\prime}}$ is the TDCS symbol duration including GI duration. The maximum CS that can be applied in CCSK is restricted by the number of subband $\left(N_{\mathrm{FFT}}\right)$. Unlike PAM, CCSK has the tendency of lower probability of error as the CS increases $[10,11]$, hence the optimum constellation size for TDCS with CCSK modulation is equal to the number of subbands $\left(N_{\mathrm{FFT}}\right)$. Theoretically it will give a better bit error rate (BER) performance and also a higher bitrate. The outputs of the random phase modules at the transmitter and at the receiver are the same. At the receiver the random phases vector with the size of $N_{\mathrm{FFT}}$ will be used for data detection. A modification to the receiver block diagram according to [12] has been made in our system. Channel estimation module is added as depicted in Fig. 2 to remove the effect of the fading. After the GI removal, the fading effect is cancelled in frequency domain, after the FFT module. The signal is transformed again into time domain by IFFT, and after addition of GI the signal is decorrelated with reference signal $c(t)$. Decorrelation for symbol demapping is simplified in [11] by choosing the maximum of absolute value of inverse Fourier transform of the product of the conjugate reference signal and received signal in frequency domain. 


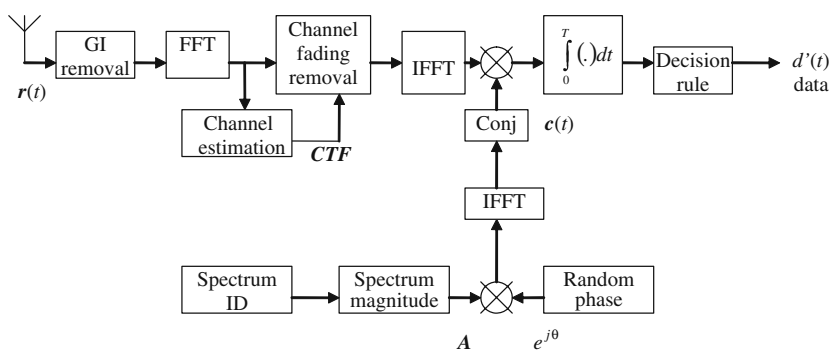

Fig. 2 Receiver architecture of TDCS with channel estimation module

\section{Orthogonal frequency division multiplexing and spectrum pooling}

In CR multi carrier OFDM based system interference avoidance is accomplished by not transmitting data and put zeros on the carriers on and adjacent to the occupied LU's band that makes the CR lose some resources to transmit data. The OFDM based CR architecture is shown in Fig. 3. The information about the occupied LU's band is provided by the spectrum magnitude module. In our AAF project this information is derived from the common control channel [8]. Unlike in TDCS system where deactivating more subband adjacent to the LU's band doesn't change the bit rate, in OFDM the bit rate will be reduced. Windowing is applied to avoid more carriers to be deactivated adjacent to LU's band. According to [4] applying window to reduce the sidelobes in the LU's band without affecting the CR's transmitted data requires two times OFDM symbol periods, and as the consequence the bit rate is becoming about half of the conventional OFDM transmission system. The bit rate of OFDM based CR system with windowing becomes:

$R_{\mathrm{OFDM}}=\frac{(\% B) \cdot N_{\mathrm{FFT}} \cdot \log _{2} \mathrm{CS}}{2 T_{s}^{\prime}}$,

where $(\% B)$ is the percentage of the carriers used to transmit data symbol. By comparing Eqs. 2 and 3 we get

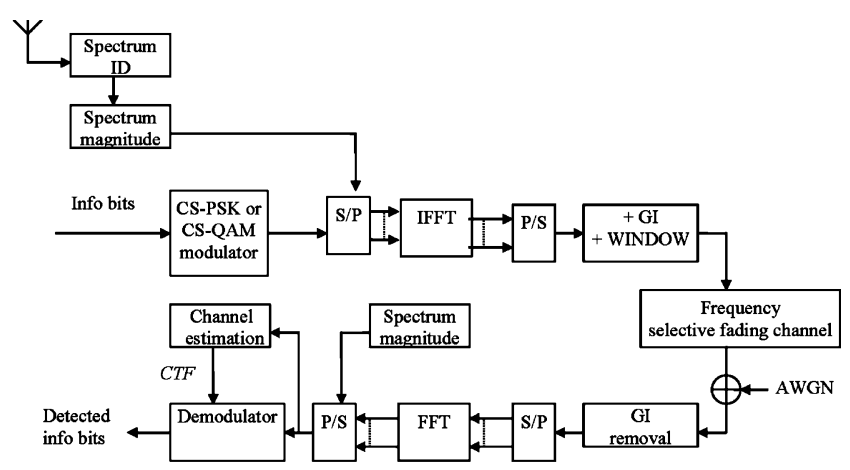

Fig. 3 Multi carrier OFDM system with interference avoidance capability that the OFDM based CR outperforms about $(\% B) . N_{\mathrm{FFT}} / 2$ times bitrate compared to TDCS based CR with PAM modulation. In the case of achieving of lowest BER as possible, BPSK is the suitable modulation mode for OFDM, while TDCS applies $N_{\text {FFT }}$ CCSK modulation. Although the bitrate of TDCS will be improved, but still the CR OFDM outperforms about $(\% B) . N_{\mathrm{FFT}} / 2 \log _{2}\left(N_{\mathrm{FFT}}\right)$ times bitrate. As the number of subband increases the OFDM bitrate gain increases exponentially.

In [4] adaptive bit loading is added in order to attain target rate by allocating the bits intelligently while the BER is minimized. In this article the application of adaptive bit loading to OFDM will not be considered in the simulations, and it is assumed that the number of deactivated carriers is sufficient enough to make the sidelobes of the spectrum very low, hence the interference to $\mathrm{LU}$ is negligible.

\section{V-BLAST architecture and algorithm}

V-BLAST is one of the promising realization of multiple input and multiple output (MIMO) system [7]. At each symbol it detects the strongest layer of the transmitted signal, cancels the effects of this strongest layer from each of the received signals, then continue to detect the strongest on the remaining layers, and so on. The MIMO transmitter model for CR TDCS and OFDM based system are depicted in Fig. 4. At the receiver side of either TDCS or OFDM, the received signals on each receiver antenna are transformed first into frequency domain. V-BLAST algorithms will
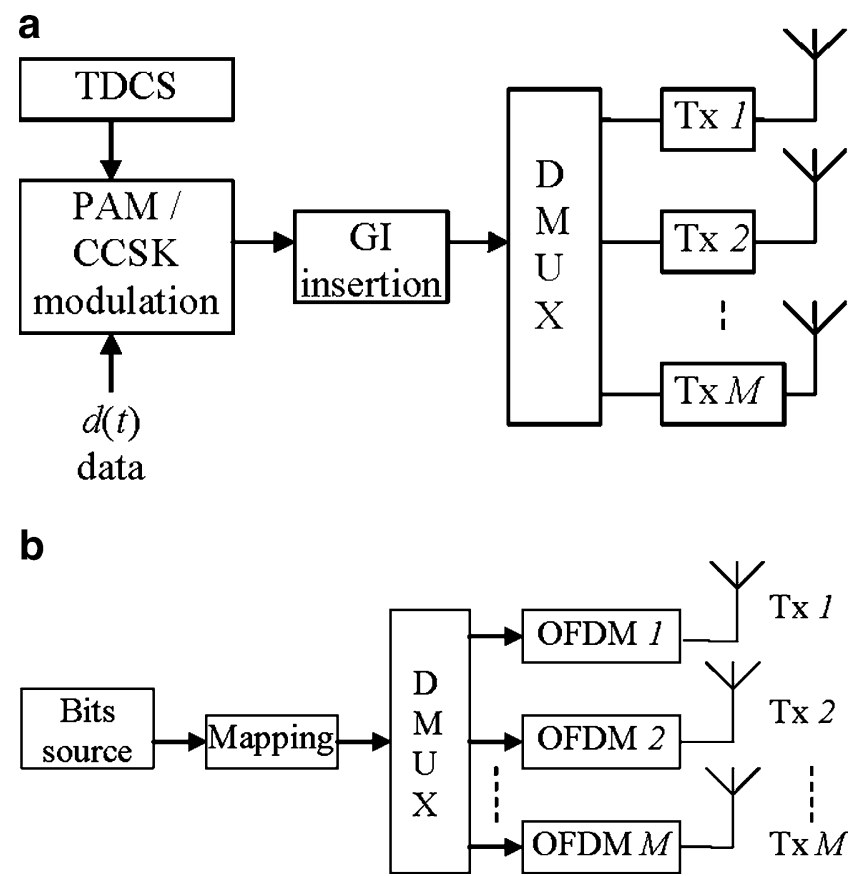

Fig. 4 MIMO transmitter architecture for CR a TDCS and b OFDM based system 
Table 1 Simulation parameters

\begin{tabular}{ll}
\hline Parameters & Values \\
\hline OFDM/TDCS parameters & \\
Carrier frequency & $5.5 \mathrm{GHz}$ \\
Number of subcarriers & 128 \\
Carrier spacing & $31.25 \mathrm{kHz}$ \\
Guard interval duration & $5.25 \mu \mathrm{s}$ \\
Channel coding & OFF \\
Channel parameters & \\
Maximum channel delay & $5 \mu \mathrm{s}$ \\
Number of taps & 6 \\
Distance between taps & $1 \mu \mathrm{s}$ \\
Number of paths & 12 \\
Power decay between taps & $1 \mathrm{~dB}$ \\
Vehicular speed & $100 \mathrm{~km} / \mathrm{h}$ \\
Fading model & Rayleigh Fading
\end{tabular}

reconstruct the transmitted signal by removing the fading effect of the channel in frequency domain. The received signals at each receiver antenna $n$ with from each of the transmit antenna $m$ is:

$Y_{n}=\sum_{m=1}^{M} H_{n m} X_{m}+\eta_{n}$,

or in matrix form the received signal vector is:

$\mathbf{Y}=\mathbf{H X}+\eta$,

where $\mathbf{Y}=\left[y_{1}, y_{2}, \ldots y_{N}\right]$ is the received signal vector, $\mathbf{X}=$ $\left[x_{1}, x_{2}, \ldots x_{M}\right]$ is the transmitted signal vector, $M$ is the number of transmit antenna, $N$ is the number of receive antenna, $\eta=\left[\eta_{1}, \eta_{2}, \ldots \eta_{N}\right]$ is the noise vector and the channel matrix $\mathbf{H}$ is described as Eq. 6 where $H_{m n}$ is the channel link between transmit antenna $m$ and receive antenna $n$ [13].

$\left[\begin{array}{cccc}H_{11} & H_{12} & \cdots & H_{1 M} \\ H_{21} & H_{n m} & \cdots & H_{2 M} \\ \vdots & \vdots & \ddots & \vdots \\ H_{N 1} & H_{N 2} & \cdots & H_{N M}\end{array}\right]$

By assuming that the receiver has the information of the channel matrix $\mathbf{H}$, the V-BLAST algorithm is implemented as follows [13]:

1. Build a Moore pseudo inverse matrix of $\mathbf{H}$,

$$
\mathbf{G}=\mathbf{H}^{+}=\left(\mathbf{H}^{*} \mathbf{H}\right)^{-1} \mathbf{H}^{*}
$$

2. Find the row of $\mathbf{G}$ where its Euclidean norm is the smallest one,

$$
k=\arg \min _{j}\left\|G_{j}\right\|
$$

and $j$ is the column of matrix G. For the TDCS system the sum is applied for $N_{\mathrm{FFT}}$ samples since one transmitted symbol is represented by $N_{\mathrm{FFT}}$ samples.

3. Take the row $k$ of $\mathbf{G}$ as the nulling vector $\mathbf{w}$,

$$
\mathbf{w}=(\mathbf{G})_{k}
$$

4. Obtain the strongest transmit signal,

$$
r_{k}=\mathbf{w}^{*} \mathbf{y}
$$

for the TDCS system $r_{k}$ will be a vector of size $N_{\mathrm{FFT}}$.

5. The estimated value of the transmitted symbol $\widehat{s}_{k}$ is derived by PSK or QAM demapping of $r_{k}$ for OFDM system while for TDCS the vector $\mathbf{r}_{k}$ will be decorrelated by the reference signal.

6. After detection of the strongest transmitted signal, its effect must be cancelled from the received signal vector to reduce the detection complexity of the remaining transmit signals, the symbol $\widehat{s}_{k}$ is mapped again by PSK or QAM mapping for OFDM while in TDCS $\widehat{s}_{k}$ is mapped into its frequency domain representation,

$$
\boldsymbol{y}=\boldsymbol{y}-(\mathbf{H})_{k}^{*} \operatorname{Mapping}\left(\widehat{s}_{k}\right)
$$

where here $k$ is the column index. The $k$-th column of channel matrix $\mathbf{H}$ is then zeroed for the purpose of detection of the strongest transmitted signal on the next layer. The algorithm returns to step 1 until transmitted symbols on all layers are detected.

\section{Simulation results}

The channel model is based on statistical discrete time model wide sense stationary uncorrelated scattering (WSSUS) [14]. The simulation parameters are provided in Table 1. The constellation size for OFDM is 2 since it gives the best theoretical probability of error. According to [13] and [14] the lowest theoretical probability of error of PAM is achieved if constellation size of 2 is chosen, while in CCSK the higher the constellation size the better is the

Table 2 Bit rate comparison

\begin{tabular}{lccc}
\hline$N_{\text {Tx }}$ & \multicolumn{2}{c}{$R_{\text {TDCS }}(\mathrm{kbps})$} & \\
\cline { 2 - 3 } & 2 PAM & & $R_{\text {OFDM }}(\mathrm{Mbps})$ \\
\hline 1 & 26.8 & 187.6 & \\
2 & 53.6 & 375.2 & 2.362 \\
3 & 80.4 & 562.8 & 4.724 \\
\hline
\end{tabular}




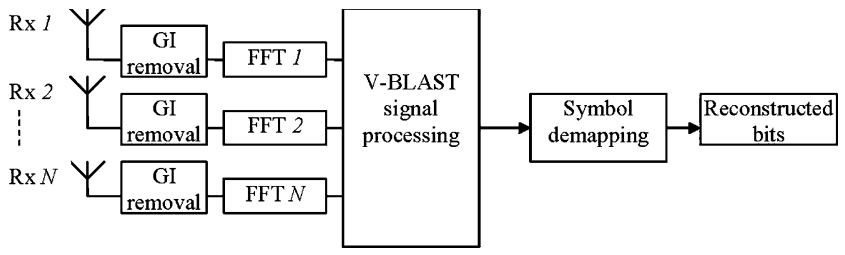

Fig. 5 MIMO receiver architecture for CR TDCS and OFDM based system

probability of error. In this article 2 PAM and 128 CCSK modulation modes are considered for TDCS simulations. Constellation size of 128 is chosen due to the restriction of number of subbands (subcarriers) in OFDM to be compared with TDCS. It is assumed that the channel transfer function (CTF) for all the links between transmit and receive antennas are perfectly known at the receiver and the synchronization is to be perfect.

According to simulation parameters in Table 1 the fully loaded signal bandwidth is $4 \mathrm{MHz}$. The channel delay spread is $1.6282 \mu$ s which makes the channel coherence bandwidth is about $123 \mathrm{kHz}$ when the envelope correlation function is more than $50 \%$. The $31.25 \mathrm{kHz}$ subcarrier spacing makes sure that each subband (carrier) experiences flat fading. LU's band is located in the middle of the RU's band, occupying 26 carriers which is equal to bandwidth of $812.5 \mathrm{kHz}$. Since in this article the effect of windowing will not be explored, the number of deactivated subband is chosen to be $40(1.25 \mathrm{MHz})$ so that the sidelobes to the LU's band and also interference from LUs to RU are negligible. Due to so many deactivated carriers, it is not necessary to transmit an OFDM symbol with period of two times the useful symbol duration. The bandwidth used by the CR system is $68.75 \%$ of fully loaded bandwidth. The bit rates of CR TDCS based with 2 PAM and 128 CCSK, and OFDM with BPSK symbol with different number of transmit antennas are given in Table 2.

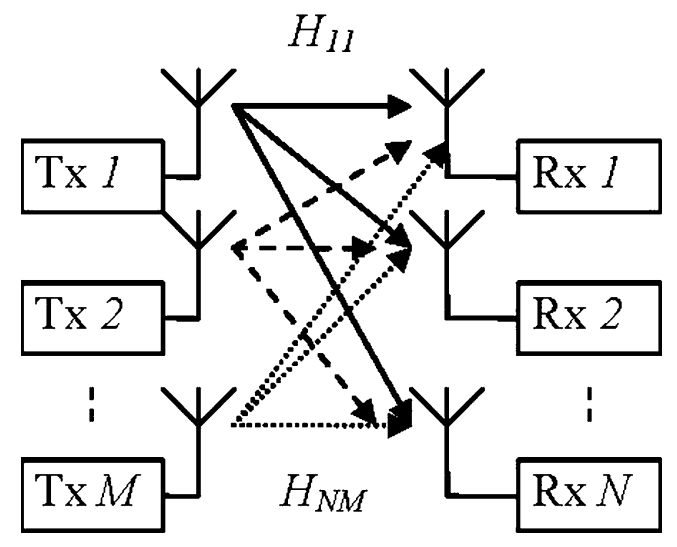

Fig. 6 MIMO channel model

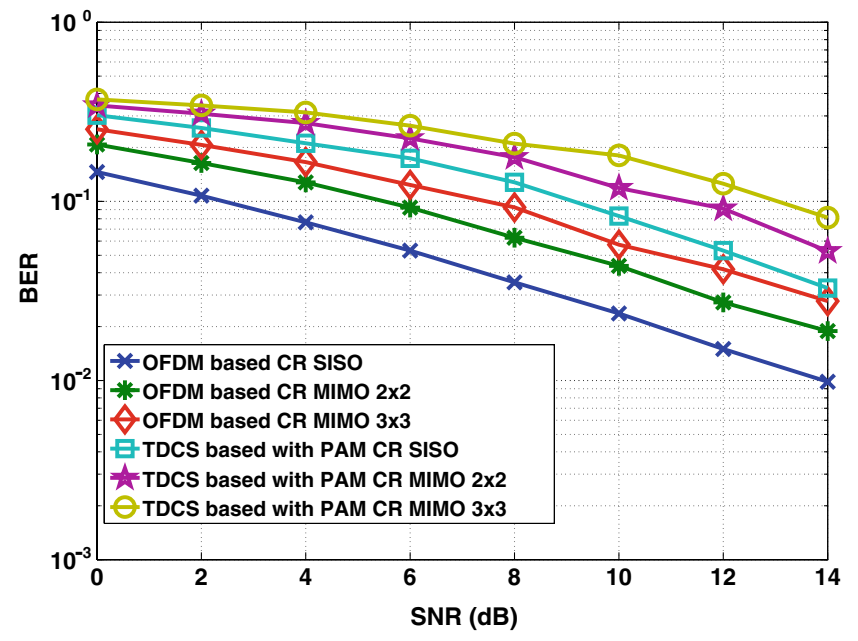

Fig. 7 BER vs SNR (decibels) performance of CR TDCS with PAM, and OFDM based with BPSK, in MIMO V-BLAST architecture with balanced antenna design

The table is derived from the same frequency selectivity resolution of TDCS and OFDM (same subband spacing). The total power for single input single output (SISO) system and MIMO system are equal. The total power for the MIMO system is divided equally among the number of the transmit antennas. Data transmitted from one transmit antenna are different from other transmit antennas (Figs. 5 and 6).

Figures 7 and 8 show that CR with OFDM consistently outperforms the CR with TDCS architecture by more than 5-6 dB signal to noise ratio (SNR) per bit gain either for single transmit and receive antenna or MIMO system with balanced design of transmit and receive antennas. BER

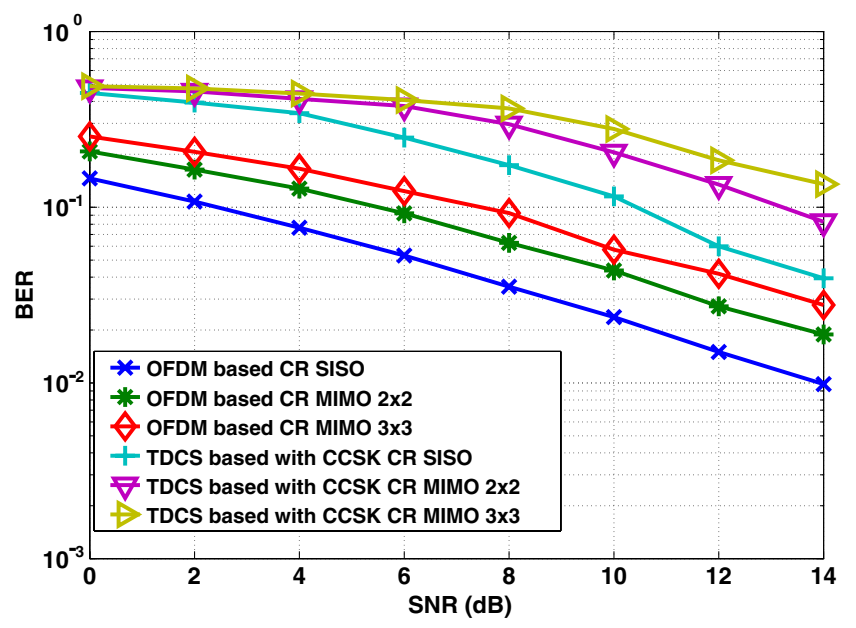

Fig. 8 BER vs SNR (decibels) performance of CR TDCS with CCSK, and OFDM based with BPSK, in MIMO V-BLAST architecture with balanced antenna design 


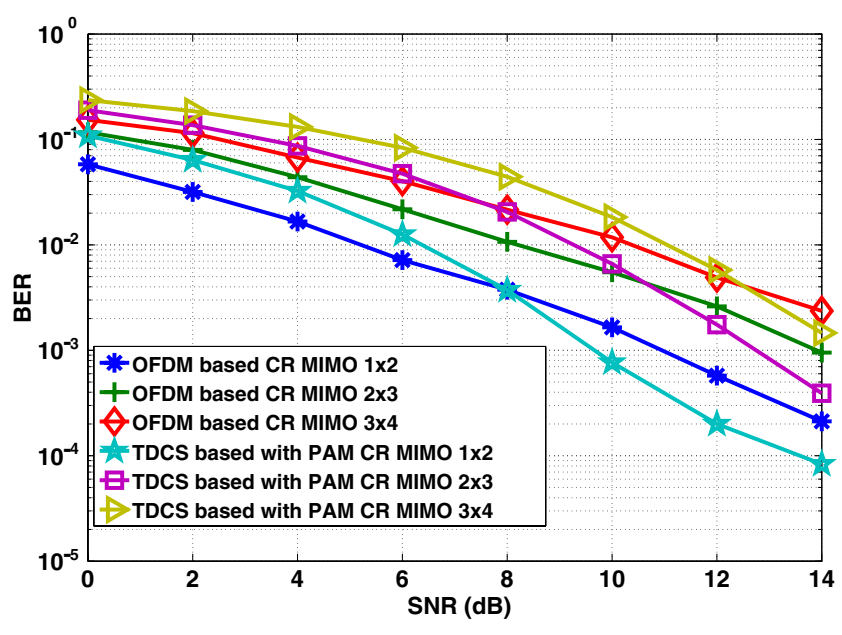

Fig. 9 BER vs SNR (decibels) performance of CR TDCS with PAM, OFDM based with BPSK, in MIMO V-BLAST architecture and 1 number different between $\mathrm{Tx}$ and $\mathrm{Rx}$ antennas

degradation as the number of transmit antenna increases with balanced design of transmit and receive antennas is compensated by the increase of the bit rate. These figures also denote that in MIMO with balanced antenna design the TDCS with PAM outperforms the TDCS with CCSK. In SISO system TDCS with PAM requires less than $10 \mathrm{~dB}$ SNR per bit to obtain BER 0.1 while TDCS with CCSK requires more than $10 \mathrm{~dB}$ SNR.

Figures 9, 10, 11 and 12 show how the BER of TDCS based CR improves its performance even outperforms the OFDM based as the number of receive antenna and SNR are increased. The SNR gain of TDCS achieved is more significant compared to OFDM system as the number of receive antennas increases while the number of transmit

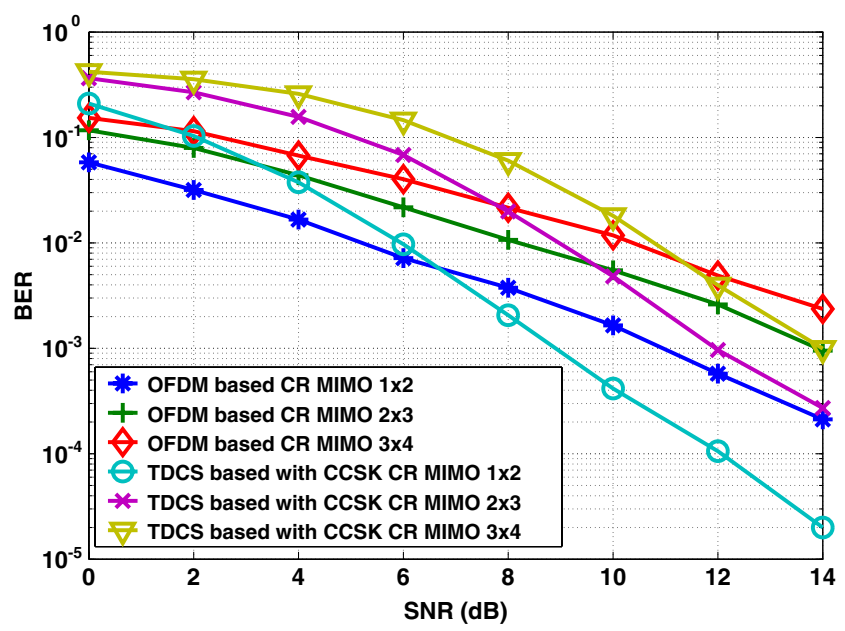

Fig. 10 BER vs SNR (decibels) performance of CR TDCS with CCSK, OFDM based with BPSK, in MIMO V-BLAST architecture and 1 number different between $\mathrm{Tx}$ and $\mathrm{Rx}$ antennas

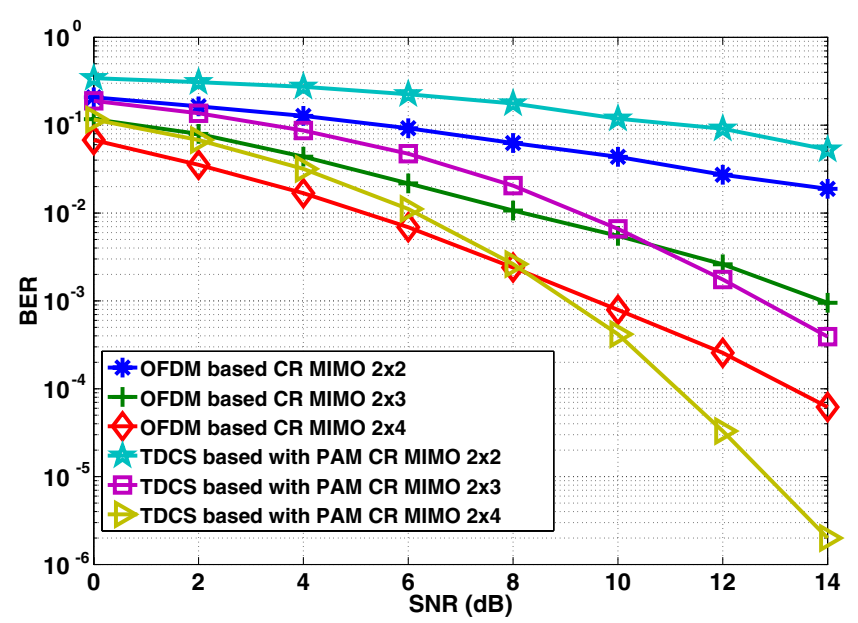

Fig. 11 BER vs SNR (decibels) performance of CR TDCS with PAM, OFDM based with BPSK in MIMO V-BLAST architecture with two Tx antennas and different number of Rx antennas

antennas remains. This gain is obtained particularly for CCSK modulation. Different with balanced antenna design as the number of receive antenna increases, at high SNR TDCS with CCSK outperforms the TDCS with PAM.

In OFDM system adding 1 more receive antenna from a balanced design $1 \times 1,2 \times 2$, or $3 \times 3$ will gain about $6 \mathrm{~dB}$ $\mathrm{SNR}$, while adding two receive antenna from the balanced design will gain about $9 \mathrm{~dB}$. Take examples at BER 0.1, OFDM with $1 \times 1,2 \times 2$ and $3 \times 3$ antennas require 2,6 and $8 \mathrm{~dB}$ SNR while for $1 \times 2,2 \times 3$ and $3 \times 4$ antennas require less than zero, 0 and $2 \mathrm{~dB}$ SNR. Figures 11 and 12 show at BER 0.01 OFDM with MIMO $2 \times 2$ requires $14 \mathrm{~dB}$ SNR, MIMO $2 \times 3$ requires $8 \mathrm{~dB}$ SNR while MIMO $2 \times 4$ requires $5 \mathrm{~dB}$ SNR. The BER vs SNR graph shows the tendency

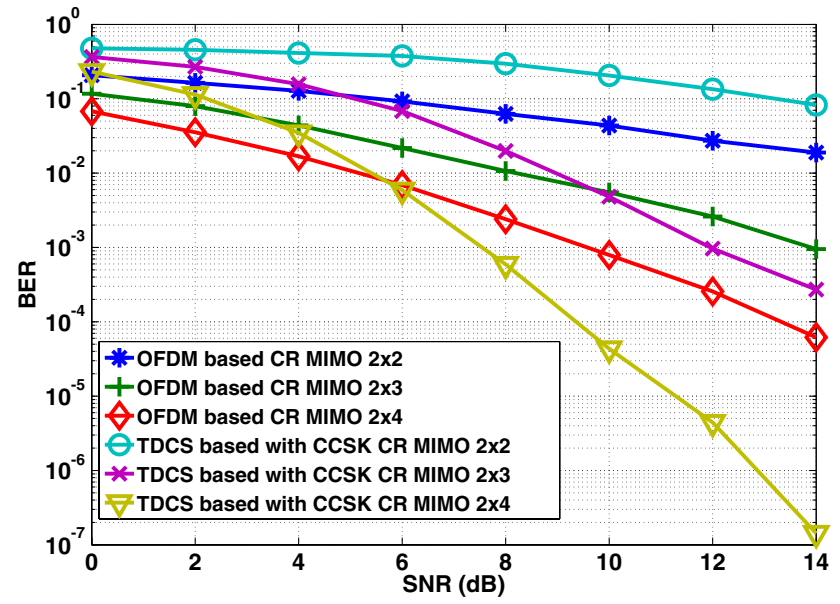

Fig. 12 BER vs SNR (decibels) performance of CR TDCS with CCSK, OFDM based with BPSK in MIMO V-BLAST architecture with two Tx antennas and different number of Rx antennas 
that the SNR gain increment is decreasing as more receive antennas are added.

In terms of complexity TDCS with CCSK requires more computation. In order to reconstruct 1 transmitted data symbol $N_{\mathrm{FFT}} \times N_{\mathrm{FFT}}$ multiplication between the received signals and the shifted reference signals are required, while TDCS with $M$ PAM requires $M \times N_{\mathrm{FFT}}$ multiplication between the received signals and reference signals. OFDM requires $M$ Euclidean distance calculation to reconstruct one transmitted symbol. In TDCS the reconstructed data decision is based on the highest correlation value, while OFDM is based on the smallest Euclidean distance.

By comparing the SNR gain of the MIMO V-BLAST with unbalanced antenna design obtained, with the $5 \mathrm{~dB}$ gain of adding adaptive bit loading subcarrierwise with Fischer algorithm to OFDM based CR SISO system in [4], we can conclude that applying MIMO $1 \times 2,2 \times 3$ or $3 \times 4$ is preferred. Not only better SNR gain performance, the schemes also increase data rate (for MIMO $2 \times 3$ and $3 \times$ 4). The trade off is the complexity of iterative algorithm on deciding the allocation bit to each subcarrier based on the SNR per carrier and channel fading per carrier for the OFDM CR SISO with adaptive bit loading, while in MIMO the channel fading on each link between transmit and receive antennas have to be estimated (more pilots required). Another disadvantage of applying adaptive bit loading is that the receiver requires side information about modulation size on each carrier. This information is transmitted in signaling symbols which reduces the data rate.

\section{Conclusions}

Single carrier with TDCS and multi carrier OFDM transmission with interference avoidance capability for Cognitive Radio system have been evaluated. Although TDCS gives larger degree of freedom in shaping its pulse without reducing its bitrate, still in SISO and MIMO with balanced number of antenna, multi carrier OFDM with interference avoidance capability is the preferred method in terms of higher bit rate, more than 5-6 dB SNR gain, and less complexity comparing to TDCS.

Problem in losing resources due to many carriers to be deactivated can be counteracted by applying multiple antenna on the transmitter. Further SNR gain can be achieved by having more antenna on the receiver than in the transmitter. TDCS with CCSK is an interesting alternative to be applied in CR system since the BER performance at high SNR can be improved tremendously as the number of receive antenna is increased while the number of transmit antenna is kept fixed. TDCS BER performance at large SNRs even outperforms multicarrier OFDM in the case when more receive than transmit antennas are used.

MIMO OFDM with higher number of receive antennas compared to transmit antennas is considered to be more interesting to be applied in the CR system compared to the application of adaptive bit loading and windowing to OFDM, as with this type of MIMO the SNR gain is higher, the bitrate can be increased and complexity of bit allocation and bit rate loss due to the increased symbol duration and signaling symbol requirement can be avoided. As the trade off the MIMO OFDM system has to estimate the channel transfer function on each antenna link which requires more pilots.

Acknowledgement The authors acknowledge the support of Dutch AAF Freeband in this research. This article is dedicated to the memory of the first author's father Baik Ukurta Purba.

Open Access This article is distributed under the terms of the Creative Commons Attribution Noncommercial License which permits any noncommercial use, distribution, and reproduction in any medium, provided the original author(s) and source are credited.

\section{References}

1. Brodersen RW et al. (2004) CORVUS: a cognitive radio approach for usage of virtual unlicensed spectrum. White Paper, Dept of Electrical Engineering and Computer Science University of Berkeley, July, 2004. http://bwrc.eecs.berkeley.edu/Research/ MCMA/CR_White_paper_finall.pdf

2. Haykin S (2005) Cognitive radio: brain empowered wireless communications. IEEE J Sel Areas Commun 2(23):201-220 Feb

3. Weiss $T$ et al (2004) Mutual interference in OFDM-based spectrum pooling systems. IEEE Veh Technol Conf, Milan, Italy, 17-19 May, CD-ROM

4. Budiarjo I, Nikookar H, Ligthart LP (2006) Combined spectrum pooling and adaptive bit loading for cognitive radio OFDM based system. 13th IEEE Symposium on Communications and Vehicular Technology, Liege, Belgium, Nov

5. Budiarjo I, Nikookar H, Ligthart LP (2006) Performance evaluation of adaptive bit loading for OFDM with channel estimation using $2 \times 1-D$ Wiener Filter. 9th International Symposium on Wireless Personal Multimedia Communications, San Diego, USA, Sept

6. Chakravarthy VD et al (2005) Cognitive radio-an adaptive waveform with spectral sharing capability. Wireless Communications and Networking Conference, IEEE 2:724-729, March

7. Wolniansky PW et al (1998) V-BLAST: an architecture for realizing very high data rates over the rich scattering wireless channel. In Proc. ISSE, Pisa, Italy, pp 295-300, Oct

8. Budiarjo I et al (2006) D3.31 radio system: first phase verification and advances in architecture and algorithms. Adaptive Ad-hoc Freeband (AAF) wireless communications, Netherlands. Sci Rep P1.0.06

9. Nikookar H, Prasad R (1997). Optimal waveform design for multicarrier transmission over a multipath channel. Proc. 47 th 
IEEE Vehicular Technology Conference, Phoenix, Arizona, USA, May 4-7, pp 1812-1816

10. Di Benedetto MG, Giancola G (2004) Understanding ultra wide band. Prentice Hall, Upper Saddle River

11. Dillard GM et al (2003) Cyclic code shift keying: a low probability of intercept communication technique. IEEE Trans Aerosp Electron Syst 39(3):786-798, July

12. Chakravarthy V et al (2005) TDCS, OFDM, and MC-CDMA: a brief tutorial. IEEE Commun Mag 43(9):S11-S16, Sept

13. de Lima ER, Flores SJ, Almenar V, Canet MJ (2004) Performance Enhancements in OFDM-WLAN systems using MIMO access techniques. International Workshop on Telecommunications, Santa Rita do Sapucai, MG, Brazil, August

14. Hoeher P (1992) A statistical discrete-time model for the WSSUS multipath channel. IEEE Trans Veh Technol 41(4):461-468, Nov

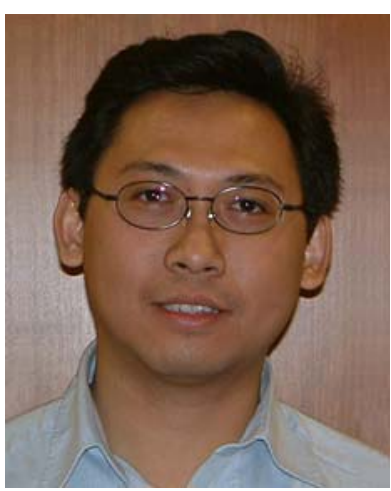

Ibrahim Budiarjo received his Bachelor of Engineering (Sarjana Teknik) degree in Electronics from Bandung Institute of Technology, Indonesia, in 2001. He got his Master of Science degree in Communications Engineering from Munich University of Technology (TU Muenchen), Germany, in 2003. He is now working toward a Ph.D. in International Research Centre for Telecommunications-Transmission and Radar, Delft University of Technology (TU Delft), The Netherlands. His research interests focus on adaptive signal processing for cognitive radio, including CDMA, OFDM, wavelet packet modulation, MIMO systems, beamforming and embedded System for cognitive radio applications.

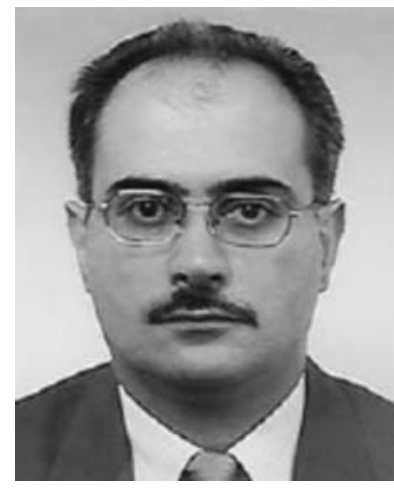

Homayoun Nikookar received his $\mathrm{Ph} . \mathrm{D}$. in Electrical Engineering from Delft University of Technology (TUDelft), The Netherlands, in 1995. From 1995 to 1998 he was a postdoc researcher at the International Research Center for TelecommunicationsTransmission and Radar, TUDelft, where since 1999 he has been an Assistant Professor. Dr. Nikookar has done research on different areas of wireless communications, including wireless channel modeling, UWB, MIMO, multicarrier transmission, Wavelet-based OFDM and CDMA. He is a senior member of the IEEE.

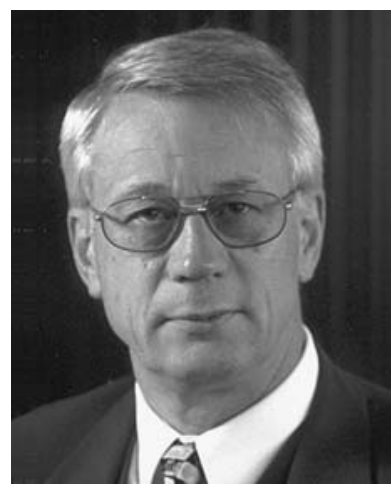

Leo P. Ligthart $\left(F^{\prime} 02\right)$ was born in Rotterdam, the Netherlands, on September 15, 1946. He received an Engineer's degree (cum laude) and a Doctor of Technology degree from Delft University of Technology in 1969 and 1985, respectively. He received Doctorates (honoris causa) at Moscow State Technical University of Civil Aviation in 1999 and Tomsk State University of Control Systems and Radioelectronics in 2001. Since 1992, he has held the chair of Microwave Transmission, Radar and Remote Sensing in the Department of Information Technology and Systems, Delft University of Technology. In 1994, he became director of the International Research Center for Telecommunications-Transmission and Radar. Prof. Ligthart's principal areas of specialization include antennas and propagation, radar and remote sensing, but he has also been active in satellite, mobile and radio communications. 